\title{
THREE RESULTS ON FROBENIUS CATEGORIES
}

\author{
XIAO-WU CHEN
}

\begin{abstract}
This paper consists of three results on Frobenius categories: (1) we give sufficient conditions on when a factor category of a Frobenius category is still a Frobenius category; (2) we show that any Frobenius category is equivalent to an extension-closed exact subcategory of the Frobenius category formed by Cohen-Macaulay modules over some additive category; this is an analogue of Gabriel-Quillen's embedding theorem for Frobenius categories; (3) we show that under certain conditions an exact category with enough projective and enough injective objects allows a natural new exact structure, with which the given category becomes Frobenius. Several applications of the results are discussed.
\end{abstract}

\section{INTRODUCTION}

Recently Ringel and Schmidmeier study intensively the classification problem in the (graded) submodule category over the truncated polynomial algebra $k[t] /\left(t^{p}\right)$ ([27]). Here, $k$ is a field and $p \geq 1$ is a natural number. This problem goes back to Birkhoff and is studied by Arnold and Simson. For an account of the history, we refer to [27. The complexity of this classification problem depends on the parameter $p$. According to $p<6, p=6$ and $p>6$, the classification problem turns out to be finite, tame and wild, respectively. We denote by $\mathcal{S}(\widetilde{p})$ the graded submodule category which is called the category of Ringel-Schmidmeier in [10]. It has a natural exact structure and becomes an exact category in the sense of Quillen; moreover, it is a Frobenius category.

In more recent work (21]), Kussin, Lenzing and Meltzer give a surprising link between the category $\mathcal{S}(\widetilde{p})$ of Ringel-Schmidmeier and the category of vector bundles on the weighted projective line of type $(2,3, p)$. To be more precise, let $\mathbb{X}$ be the weighted projective line of type $(2,3, p)$ in the sense of Geigle and Lenzing ([15]). Denote by vect $\mathbb{X}$ the category of vector bundles on $\mathbb{X}$. It has a natural exact structure such that it is a Frobenius category ([20]). Following [21] we denote by $\mathcal{F}$ the additive closure of the so-called fading line bundles. Consider the factor category vect $\mathbb{X} /[\mathcal{F}]$ of vect $\mathbb{X}$ modulo those morphisms factoring through $\mathcal{F}$. One of the main results in [21] states that there is an equivalence of categories between vect $\mathbb{X} /[\mathcal{F}]$ and $\mathcal{S}(\widetilde{p})$; also see Example 4.3. From this equivalence the authors recover some major results in [27] via certain known results on vector bundles over the weighted projective lines. Note that according to $p<6, p=6$ and $p>6$ the weighted projective line $\mathbb{X}$ is domestic, tubular and wild, respectively.

Date: October 3, 2018.

1991 Mathematics Subject Classification. 18E10, 16G50, 18E30.

Key words and phrases. Frobenius category, Cohen-Macaulay module, weighted projective line, matrix factorization, minimal monomorphism.

This project was supported by Alexander von Humboldt Stiftung and National Natural Science Foundation of China (No.10971206).

E-mail: xwchen@mail.ustc.edu.cn. 
We have noted above that the category $\mathcal{S}(\widetilde{p})$ of Ringel-Schmidmeier is Frobenius. Hence via the equivalence mentioned above one infers that the factor category vect $\mathbb{X} /[\mathcal{F}]$ is also a Frobenius category; see $[21$, Theorem A]. However a direct argument of this surprising fact seems missing. More generally, one may ask when a factor category of a Frobenius category is still Frobenius. This is one of the motivations of the present paper. Another motivation is to understand the minimal monomorphism in the sense of Ringel-Schmidmeier ([26]), which plays an important role in the study of Auslander-Reiten sequences in submodule categories.

The paper is organized as follows, which mainly consists of three results on Frobenius categories. We collect in Section 2 some basic facts and notions on exact categories and Frobenius categories. In Section 3 we give sufficient conditions on when a factor category of a Frobenius category is still Frobenius; see Theorem 3.1. We apply the obtained result to recover [21, Theorem A], modulo a certain technical fact which is somehow hidden in [21. We also apply the result to the Frobenius category of matrix factorizations. In Section 4 we prove a general result on Frobenius categories: each Frobenius category is equivalent, as exact categories, to an extension-closed exact subcategory of the Frobenius category formed by Cohen-Macaulay modules over some additive category; see Theorem 4.2. This can be viewed as an analogue of Gabriel-Quillen's embedding theorem for Frobenius categories. We observe that the category $\mathcal{S}(\widetilde{p})$ of Ringel-Schmidmeier can be viewed as the category of CohenMacaulay modules over some graded algebras ([10]). Together with this observation and the result in Section 3, our general result recovers a part of [21, Theorem C]. In Section 5 we give sufficient conditions such that on an exact category with enough projective and enough injective objects there exists another natural exact structure, with which the given category becomes Frobenius; see Theorem 5.1. An application of this result allows us to interpret the minimal monomorphism operation in [26] as a triangle functor, which is right adjoint to an inclusion triangle functor.

\section{Preliminaries on exaCt CATEgories}

In this section we collect some basic facts and notions on exact categories and Frobenius categories. The basic reference is [18, Appendix A]. For a systematical treatment of exact category, we refer to [8].

Let $\mathcal{A}$ be an additive category. A composable pair of morphisms is a sequence $X \stackrel{i}{\rightarrow} Y \stackrel{d}{\rightarrow} Z$; such a composable pair is denoted by $(i, d)$. Two composable pairs $(i, d)$ and $\left(i^{\prime}, d^{\prime}\right)$ are isomorphic provided that there are isomorphisms $f: X \rightarrow X^{\prime}$, $g: Y \rightarrow Y^{\prime}$ and $h: Z \rightarrow Z^{\prime}$ such that $g \circ i=i^{\prime} \circ f$ and $h \circ d=d^{\prime} \circ g$. A composable pair $(i, d)$ is called a kernel-cokernel pair provided that $i=\operatorname{Ker} d$ and $d=$ Cok $i$.

An exact structure on an additive category $\mathcal{A}$ is a chosen class $\mathcal{E}$ of kernel-cokernel pairs in $\mathcal{A}$, which is closed under isomorphisms and is subject to the following axioms $(\mathrm{Ex} 0),(\mathrm{Ex} 1),(\mathrm{Ex} 1)^{\mathrm{op}},(\mathrm{Ex} 2)$ and $(\mathrm{Ex} 2)^{\mathrm{op}}$. A pair $(i, d)$ in the chosen class $\mathcal{E}$ is called a conflation, while $i$ is called an inflation and $d$ is called a deflation. The pair $(\mathcal{A}, \mathcal{E})$ is called an exact category in the sense of Quillen ([25]); sometimes we suppress the class $\mathcal{E}$ and just say that $\mathcal{A}$ is an exact category.

Following [18, Appendix A], the axioms of exact category are listed as follows:

(Ex0) the identity morphism of the zero object is a deflation;

(Ex1) a composition of two deflations is a deflation;

$(\mathrm{Ex} 1)^{\mathrm{op}}$ a composition of two inflations is an inflation; 
(Ex2) for a deflation $d: Y \rightarrow Z$ and a morphism $f: Z^{\prime} \rightarrow Z$ there exists a pullback diagram such that $d^{\prime}$ is a deflation:

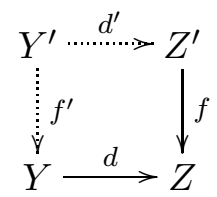

$\left(\right.$ Ex2) ${ }^{\text {op }}$ for an inflation $i: X \rightarrow Y$ and a morphism $f: X \rightarrow X^{\prime}$ there exists a pushout diagram such that $i^{\prime}$ is an inflation:

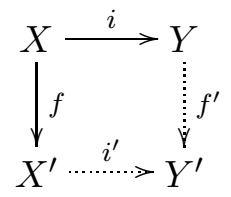

Let us remark that the axiom $(\mathrm{Ex} 1)^{\text {op }}$ can be deduced from the other axioms; see [18, Appendix A].

For an exact category $\mathcal{A}$, a full additive subcategory $\mathcal{B} \subseteq \mathcal{A}$ is said to be extensionclosed provided that for any conflation $X \stackrel{i}{\rightarrow} Y \stackrel{d}{\rightarrow} Z$ with $X, Z \in \mathcal{B}$ we have $Y \in \mathcal{B}$. In this case, the subcategory $\mathcal{B}$ inherits the exact structure from $\mathcal{A}$ to become an exact category. We will call such a subcategory an extension-closed exact subcategory. Observe that any abelian category has a natural exact structure such that conflations are induced by short exact sequences. Consequently, any full additive subcategory in an abelian category which is closed under extensions has a natural exact structure and then becomes an exact category.

Recall that an additive functor $F: \mathcal{B} \rightarrow \mathcal{A}$ between two exact categories is called exact provided that it sends conflations to conflations; an exact functor $F: \mathcal{B} \rightarrow \mathcal{A}$ is said to be an equivalence of exact categories provided that $F$ is an equivalence and there exists a quasi-inverse of $F$ which is exact.

From now on $\mathcal{A}$ is an exact category. We will need the following two facts. For the first fact, we refer to the first step in the proof of [18, Proposition A.1]; for the second one, we refer to the axiom c) in the proof of [18, Proposition A.1]

Lemma 2.1. Consider the diagram in (Ex2). Then the sequence

$$
Y^{\prime} \stackrel{\left(\begin{array}{c}
d^{\prime} \\
-f^{\prime}
\end{array}\right)}{\longrightarrow} Z^{\prime} \oplus Y \stackrel{(f, d)}{\longrightarrow} Z
$$

is a conflation and we have a commutative diagram such that the two rows are conflations:

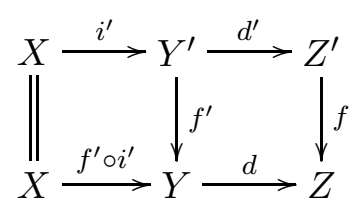

Lemma 2.2. Let $d$ be a morphism such that $d \circ e$ is a deflation for some morphism e. Assume further that d has a kernel. Then $d$ is a deflation.

Recall that an object $P$ in $\mathcal{A}$ is projective provided that the functor $\operatorname{Hom}_{\mathcal{A}}(P,-)$ sends conflations to short exact sequences; this is equivalent to that any deflation ending at $P$ splits. The exact category $\mathcal{A}$ is said to have enough projective objects 
provided that each object $X$ fits into a deflation $d: P \rightarrow X$ with $P$ projective. Dually one has the notions of injective object and having enough injective objects.

An exact category $\mathcal{A}$ is said to be Frobenius provided that it has enough projective and enough injective objects, and the class of projective objects coincides with the class of injective objects ([17, Section 3]). The importance of Frobenius categories lies in that they give rise naturally to triangulated categories; see [16] and [18, 1.2].

The following notion will be convenient for us: for a Frobenius category $\mathcal{A}$, an extension-closed exact subcategory $\mathcal{B} \subseteq \mathcal{A}$ is said to be admissible provided that each object $B$ in $\mathcal{B}$ fits into conflations $B \rightarrow P \rightarrow B^{\prime}$ and $B^{\prime \prime} \rightarrow Q \rightarrow B$ in $\mathcal{B}$ such that $P, Q$ are projective in $\mathcal{A}$. Note that an admissible subcategory $\mathcal{B}$ of a Frobenius category $\mathcal{A}$ is still Frobenius; moreover, an object $B$ in $\mathcal{B}$ is projective if and only if it is projective viewed as an object in $\mathcal{A}$.

\section{FACtor CAtegory of Frobenius CAtegory}

In this section we study a certain factor category of a Frobenius category. We give sufficient conditions on when the factor category inherits the exact structure from the given Frobenius category such that it becomes a Frobenius category. As an application, our result specializes to [21, Theorem A] modulo certain technical results which are somehow hidden in [21. We give an example to apply our result to the category of matrix factorizations ([12]).

Let $(\mathcal{A}, \mathcal{E})$ be a Frobenius category. Denote by $\mathcal{P}$ the full subcategory consisting of projective objects. Let $\mathcal{F} \subseteq \mathcal{P}$ be a full additive subcategory. For two objects $X, Y$ in $\mathcal{A}$ denote by $[\mathcal{F}](X, Y)$ the subgroup of $\operatorname{Hom}_{\mathcal{A}}(X, Y)$ consisting of those morphisms which factor through an object in $\mathcal{F}$. Denote by $\mathcal{A} /[\mathcal{F}]$ the factor category of $\mathcal{A}$ modulo $\mathcal{F}$ : the objects are the same as the ones in $\mathcal{A}$, for two objects $X$ and $Y$ the Hom space is given by the quotient group $\operatorname{Hom}_{\mathcal{A}}(X, Y) /[\mathcal{F}](X, Y)$ and the composition is induced by the one in $\mathcal{A}$; compare [3, p.101]. Note that the factor category $\mathcal{A} /[\mathcal{F}]$ is an additive category.

Denote by $\pi_{\mathcal{F}}: \mathcal{A} \rightarrow \mathcal{A} /[\mathcal{F}]$ the canonical functor. Denote by $\mathcal{E}_{\mathcal{F}}$ the class of composable pairs in $\mathcal{A} /[\mathcal{F}]$ which are isomorphic to composable pairs $\left(\pi_{\mathcal{F}}(i), \pi_{\mathcal{F}}(d)\right)$ for $(i, d) \in \mathcal{E}$.

The case $\mathcal{F}=\mathcal{P}$ is of particular interest, since the corresponding factor category, known as the stable category of $\mathcal{A}$ and denoted by $\underline{\mathcal{A}}$, has a natural triangulated structure. In this case the canonical functor $\pi_{\mathcal{P}}: \mathcal{A} \rightarrow \underline{\mathcal{A}}$ sends conflations to exact triangles. For details, see [16, Chapter I, Section 2].

We are interested in the following question: when the factor category $\mathcal{A} /[\mathcal{F}]$ becomes a Frobenius category such that its exact structure is given by $\mathcal{E}_{\mathcal{F}}$ ? Note that in general the case $\mathcal{F}=\mathcal{P}$ will not meet the requirement. The aim of this section is to give a partial answer to this question.

Recall that a pseudo-kernel of a morphism $f: X \rightarrow Y$ is a morphism $c: Y \rightarrow C$ such that $c \circ f=0$ and it satisfies that any morphism $c^{\prime}: Y \rightarrow C^{\prime}$ with $c^{\prime} \circ f=0$ factors through $c$. Dually one has the notion of pseudo-cokernel; see [4, Section 2].

Recall that for a subcategory $\mathcal{S}$ of $\mathcal{A}$, a morphism $f: S \rightarrow X$ is said to be a right $\mathcal{S}$-approximation of $X$ provided that $S \in \mathcal{S}$ and any morphism from an object in $\mathcal{S}$ to $X$ factors through $f$. Dually one has the notion of left $\mathcal{S}$-approximation; see [2, Section 1]. 
Our first result is as follows, which gives sufficient conditions on when the pair $\left(\mathcal{A} /[\mathcal{F}], \mathcal{E}_{\mathcal{F}}\right)$ is a Frobenius category.

Theorem 3.1. Let $(\mathcal{A}, \mathcal{E})$ be a Frobenius category and let $\mathcal{P}$ denote the subcategory of projective objects. Suppose that $\mathcal{F} \subseteq \mathcal{P}$ satisfies the following conditions:

(1) any object $A$ in $\mathcal{A}$ fits into a sequence

$$
A \stackrel{i_{A}}{\longrightarrow} F_{A} \stackrel{p_{A}}{\longrightarrow} P_{A}
$$

such that $i_{A}$ is a left $\mathcal{F}$-approximation of $A, P_{A} \in \mathcal{P}$ and $p_{A}$ is a pseudocokernel of $i_{A}$;

(2) any object $A$ in $\mathcal{A}$ fits into a sequence

$$
P^{A} \stackrel{i^{A}}{\longrightarrow} F^{A} \stackrel{p^{A}}{\longrightarrow} A
$$

such that $p^{A}$ is a right $\mathcal{F}$-approximation of $A, P^{A} \in \mathcal{P}$ and $i^{A}$ is a pseudokernel of $p^{A}$.

Then the pair $\left(\mathcal{A} /[\mathcal{F}], \mathcal{E}_{\mathcal{F}}\right)$ is a Frobenius category.

Proof. In the proof, we write $\pi_{\mathcal{F}}$ as $\pi$. We will divide the proof into three steps.

Step 1. We will first show that the composable pairs in $\mathcal{E}_{\mathcal{F}}$ are kernel-cokernel pairs. It suffices to show that for any conflation $X \stackrel{i}{\rightarrow} Y \stackrel{d}{\rightarrow} Z$ in $\mathcal{A}$ we have $\pi(i)=\operatorname{Ker} \pi(d)$ and $\pi(d)=\operatorname{Cok} \pi(i)$. We will only show that $\pi(i)=\operatorname{Ker} \pi(d)$, and the remaining equality is shown by a dual argument.

To show that $\pi(i)$ is mono, it suffices to show that any morphism $a: A \rightarrow X$ in $\mathcal{A}$ having the property $i \circ a \in[\mathcal{F}](A, Y)$ necessarily lies in $[\mathcal{F}](A, X)$. Consider the sequence in (1) for $A$. Since $i \circ a: A \rightarrow Y$ factors through an object in $\mathcal{F}$ and $i_{A}: A \rightarrow F_{A}$ is a left $\mathcal{F}$-approximation, there is a morphism $t: F_{A} \rightarrow Y$ such that $i \circ a=t \circ i_{A}$. Using that $p_{A}$ is a pseudo-cokernel of $i_{A}$, we have a morphism $s: P_{A} \rightarrow Z$ making the following diagram commute

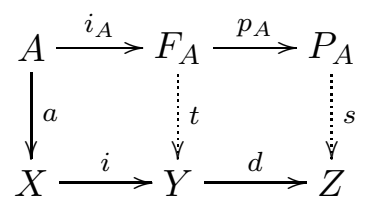

Since $P_{A}$ is projective and $(i, d)$ is a conflation, we may lift $s$ to a morphism $s^{\prime}: P_{A} \rightarrow$ $Y$ such that $d \circ s^{\prime}=s$. Then we have

$$
d \circ\left(t-s^{\prime} \circ p_{A}\right)=d \circ t-s \circ p_{A}=0 .
$$

Since $i=\operatorname{Ker} d$, there exists $a^{\prime}: F_{A} \rightarrow X$ such that $i \circ a^{\prime}=t-s^{\prime} \circ p_{A}$. Composing the two sides with $i_{A}$, we get $i \circ a^{\prime} \circ i_{A}=t \circ i_{A}=i \circ a$. Note that $i$ is mono and $F_{A} \in \mathcal{F}$. Then we have $a=a^{\prime} \circ i_{A}$ and it lies in $[\mathcal{F}](A, X)$.

Having shown that $\pi(i)$ is mono, it suffices to show that $\pi(i)$ is a pseudo-kernel of $\pi(d)$. Then we have $\pi(i)=\operatorname{Ker} \pi(d)$. For this end, take a morphism $a: A \rightarrow Y$ such that $d \circ a \in[\mathcal{F}](A, Z)$. We will show that $\pi(a)$ factors through $\pi(i)$. Assume that $d \circ a$ factors as $A \stackrel{x}{\rightarrow} F \stackrel{y}{\rightarrow} Z$ with $F \in \mathcal{F}$. Since $F$ is projective and $(i, d)$ is a conflation, we may lift $y$ to a morphism $y^{\prime}: F \rightarrow Y$ such that $d \circ y^{\prime}=y$. Then we have

$$
d \circ\left(a-y^{\prime} \circ x\right)=d \circ a-y \circ x=0 .
$$


Hence there exists a morphism $a^{\prime}: A \rightarrow X$ such that $a-y^{\prime} \circ x=i \circ a^{\prime}$. Note that $F \in \mathcal{F}$. Applying $\pi$ we get $\pi(a)=\pi(i) \circ \pi\left(a^{\prime}\right)$.

Step 2. We will show next that the pair $\left(\mathcal{A} /[\mathcal{F}], \mathcal{E}_{\mathcal{F}}\right)$ is an exact category. Note that by definition a morphism $\delta: \pi(Y) \rightarrow \pi(Z)$ is a deflation if and only if there exist morphisms $a: Y \rightarrow Y^{\prime}$ and $b: Z^{\prime} \rightarrow Z$ such that $\pi(a)$ and $\pi(b)$ are isomorphisms, and a deflation $d: Y^{\prime} \rightarrow Z^{\prime}$ in $\mathcal{A}$ such that we have a factorization $\delta=\pi(b) \circ \pi(d) \circ \pi(a)$. The axiom (Ex0) is trivial.

To show (Ex1), assume that we are given two deflations $\delta: \pi(Y) \rightarrow \pi(Z)$ and $\gamma: \pi(Z) \rightarrow \pi(W)$ in $\mathcal{A} /[\mathcal{F}]$. We may assume that $\delta$ and $\gamma$ factor as $\pi(Y) \stackrel{\pi(a)}{\rightarrow}$ $\pi\left(Y^{\prime}\right) \stackrel{\pi(d)}{\rightarrow} \pi\left(Z^{\prime}\right) \stackrel{\pi(b)}{\rightarrow} \pi(Z)$ and $\pi(Z) \stackrel{\pi(x)}{\rightarrow} \pi\left(Z^{\prime \prime}\right) \stackrel{\pi(e)}{\rightarrow} \pi\left(W^{\prime}\right) \stackrel{\pi(y)}{\rightarrow} \pi(W)$, respectively. Here $d: Y^{\prime} \rightarrow Z^{\prime}$ and $e: Z^{\prime \prime} \rightarrow W^{\prime}$ are deflations in $\mathcal{A}$. Take a morphism $z: Z^{\prime \prime} \rightarrow Z$ such that $\pi(z)=(\pi(x) \circ \pi(b))^{-1}$. By (Ex2) we have the pullback diagram in $\mathcal{A}$

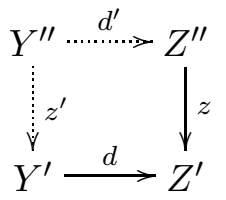

such that $d^{\prime}$ is a deflation. By Lemma 2.1 the sequence $Y^{\prime \prime} \stackrel{\left(\stackrel{d^{\prime}}{\rightarrow z^{\prime}}\right)}{\rightarrow} Z^{\prime \prime} \oplus Y^{\prime} \stackrel{(z, d)}{\rightarrow} Z^{\prime}$ is a conflation in $\mathcal{A}$. By the first step, applying $\pi$ to this sequence we get a kernel-cokernel pair in the factor category $\mathcal{A} /[\mathcal{F}]$. In particular, the diagram above is still a pullback diagram in $\mathcal{A} /[\mathcal{F}]$. Hence the fact that $\pi(z)$ is an isomorphism implies that $\pi\left(z^{\prime}\right)$ is also an isomorphism. Take a morphism $a^{\prime}: Y^{\prime} \rightarrow Y^{\prime \prime}$ in $\mathcal{A}$ such that $\pi\left(a^{\prime}\right)=\pi\left(z^{\prime}\right)^{-1}$. Then $\gamma \circ \delta$ factors as

$$
\pi(Y) \stackrel{\pi\left(a^{\prime} \circ a\right)}{\longrightarrow} \pi\left(Y^{\prime \prime}\right) \stackrel{\pi\left(e \circ d^{\prime}\right)}{\longrightarrow} \pi\left(W^{\prime}\right) \stackrel{\pi(y)}{\longrightarrow} \pi(W) .
$$

By $(\mathrm{Ex} 1) e \circ d^{\prime}$ is a deflation in $\mathcal{A}$. Observe that both $\pi\left(a^{\prime} \circ a\right)$ and $\pi(y)$ are isomorphisms. Then we have that $\gamma \circ \delta$ is a deflation in $\mathcal{A} /[\mathcal{F}]$, proving the axiom (Ex1). Dually one shows $(\mathrm{Ex} 1)^{\mathrm{op}}$.

To show (Ex2), take a deflation $\delta: \pi(Y) \rightarrow \pi(Z)$ and a morphism $\pi(f): \pi\left(Z^{\prime}\right) \rightarrow$ $\pi(Z)$. Without loss of generality we may assume that $\delta=\pi(d)$ for a deflation $d: Y \rightarrow Z$ in $\mathcal{A}$. Then we apply (Ex2) for $\mathcal{A}$ to get a pullback diagram in $\mathcal{A}$. As above, using Lemma 2.1 and the first step, the obtained diagram is also a pullback diagram in the factor category $\mathcal{A} /[\mathcal{F}]$. This proves the axiom $($ Ex2) for $\mathcal{A} /[\mathcal{F}]$. Dually one shows $(\mathrm{Ex} 2)^{\mathrm{op}}$.

Step 3. The exact category $\left(\mathcal{A} /[\mathcal{F}], \mathcal{E}_{\mathcal{F}}\right)$ is Frobenius. Recall that up to isomorphism conflations in $\mathcal{A} /[\mathcal{F}]$ are given by the images of conflations in $\mathcal{A}$. Then it follows immediately that objects in $\mathcal{P} /[\mathcal{F}]$ are projective and injective in the exact category $\left(\mathcal{A} /[\mathcal{F}], \mathcal{E}_{\mathcal{F}}\right)$; moreover, each object $\pi(X)$ in $\mathcal{A} /[\mathcal{F}]$ admits a deflation $\pi(P) \rightarrow \pi(X)$ and an inflation $\pi(X) \rightarrow \pi(I)$ with $\pi(P), \pi(I) \in \mathcal{P} /[\mathcal{F}]$. From these, one concludes immediately that the exact category $\left(\mathcal{A} /[\mathcal{F}], \mathcal{E}_{\mathcal{F}}\right)$ is Frobenius.

Remark 3.2. As shown in the third step above, the full subcategory of $\mathcal{A} /[\mathcal{F}]$ consisting of projective objects is equal to $\mathcal{P} /[\mathcal{F}]$. Using again the fact that up to isomorphism conflations in $\mathcal{A} /[\mathcal{F}]$ are given by the images of conflations in $\mathcal{A}$, we have an identification $\underline{\mathcal{A}}=\underline{\mathcal{A} /[\mathcal{F}]}$ of triangulated categories. 
We will apply Theorem 3.1 in two examples. We begin with our motivating example. We will see that, modulo certain technical results in [21, Theorem 3.1 specializes to [21, Theorem A].

Example 3.3. Let $k$ be a field and $p \geq 2$ be a natural number. Let $\mathbb{X}$ be the weighted projective line of type $(2,3, p)$ in the sense of Geigle and Lenzing ([15]). Denote by coh $\mathbb{X}$ the abelian category of coherent sheaves on $\mathbb{X}$ and by $\mathcal{O}$ the structure sheaf on $\mathbb{X}$. Denote by $L$ the rank one abelian group on three generators $\vec{x}_{1}, \overrightarrow{x_{2}}, \overrightarrow{x_{3}}$ subject to the relations $2 \vec{x}_{1}=3 \vec{x}_{2}=p \vec{x}_{3}$. Recall that the group $L$ acts on coh $\mathbb{X}$. We denote the action of an element $\vec{x} \in L$ on a sheaf $E$ by $E(\vec{x})$.

Denote by vect $\mathbb{X}$ the full subcategory of coh $\mathbb{X}$ consisting of vector bundles. Recall that all the line bundles on $\mathbb{X}$ are given by $\mathcal{O}(\vec{x})$ for $\vec{x} \in L$; moreover, $\mathcal{O}(\vec{x}) \simeq \mathcal{O}(\vec{y})$ implies that $\vec{x}=\vec{y}$. In other words, the Picard group of $\mathbb{X}$ is isomorphic to $L$; see [15, Proposition 2.1]. Recall that the subcategory vect $\mathbb{X} \subseteq$ coh $\mathbb{X}$ is closed under extensions and then it has a natural exact structure. However with this exact structure the category vect $\mathbb{X}$ is not Frobenius.

Following [20] a short exact sequence $\eta: 0 \rightarrow E^{\prime} \rightarrow E \rightarrow E^{\prime \prime} \rightarrow 0$ of vector bundles is distinguished provided that the sequences $\operatorname{Hom}(\mathcal{O}(\vec{x}), \eta)$ are exact for all $\vec{x} \in L$. By Serre duality this is equivalent to that the sequences $\operatorname{Hom}(\eta, \mathcal{O}(\vec{x}))$ are exact for all $\vec{x} \in L$. Observe that the category vect $\mathbb{X}$ of vector bundles is an exact category such that conflations are induced by distinguished short exact sequences; compare Lemma 5.2. We denote by $\mathcal{A}$ this exact category. Moreover, the exact category $\mathcal{A}$ is Frobenius such that its subcategory $\mathcal{P}$ of projective objects is equal to the additive closure of all line bundles. For details, see [20].

The following terminology is taken from [21]. A line bundle $\mathcal{O}(\vec{x})$ is said to be fading provided that $\vec{x} \notin \mathbb{Z} \vec{x}_{3} \cup \vec{x}_{2}+\mathbb{Z} \vec{x}_{3}$. Take $\mathcal{F} \subseteq \mathcal{P}$ to be the additive closure of these fading line bundles. We claim that the subcategory $\mathcal{F}$ satisfies the conditions in Theorem 3.1, Then it follows from Theorem 3.1 that the factor category $\mathcal{A} /[\mathcal{F}]$ inherits the Frobenius exact structure from the one of $\mathcal{A}$; this is [21, Theorem $\mathrm{A}]$.

In fact, the proof of [21, Proposition 3.13] yields the following technical fact: for a vector bundle $E$ there is a short exact sequence $0 \rightarrow E \stackrel{\alpha}{\rightarrow} C \rightarrow P_{1} \rightarrow 0$ with $C \in \mathcal{F}$ and $P_{1} \in \mathcal{P}$; moreover, the morphism $\alpha$ is a left $\mathcal{F}$-approximation (by [21, Lemma $3.12(2)])$. Here we are consistent in notation with the proof of [21, Proposition 3.13]. Note that one has a dual version of this result using the duality $d: \mathcal{A} \rightarrow \mathcal{A}$ in the proof of [21, Proposition 3.2].

The second example shows that a certain factor category of the category of matrix factorizations has a Frobenius exact structure.

Example 3.4. Let $R$ be a commutative noetherian ring and let $f \in R$ be a regular element. Recall that a matrix factorization of $f$ is a composable pair $P^{0} \stackrel{d_{P}^{0}}{\rightarrow} P^{1} \stackrel{d_{P}^{1}}{\rightarrow} P^{0}$ consisting of finitely generated projective $R$-modules such that $d_{P}^{1} \circ d_{P}^{0}=f \operatorname{Id}_{P^{0}}$ and $d_{P}^{0} \circ d_{P}^{1}=f \operatorname{Id}_{P^{1}}$; a morphism $\left(f^{0}, f^{1}\right):\left(d_{P}^{0}, d_{P}^{1}\right) \rightarrow\left(d_{Q}^{0}, d_{Q}^{1}\right)$ between matrix factorizations consists of two morphisms $f^{0}: P^{0} \rightarrow Q^{0}$ and $f^{1}: P^{1} \rightarrow Q^{1}$ of $R$ modules such that $d_{Q}^{0} \circ f^{0}=f^{1} \circ d_{P}^{0}$ and $d_{Q}^{1} \circ f^{1}=f^{0} \circ d_{P}^{1}$. Observe that since $f$ is regular, the two morphisms $d_{P}^{0}$ and $d_{P}^{1}$ in a matrix factorization are mono. For details, see [12, Section 5].

Denote by $\mathrm{MF}_{R}(f)$ the category of matrix factorizations of $f$. It has a natural exact structure such that a sequence $\left(d_{P^{\prime}}^{0}, d_{P^{\prime}}^{1}\right) \rightarrow\left(d_{P}^{0}, d_{P}^{1}\right) \rightarrow\left(d_{P^{\prime \prime}}^{0}, d_{P^{\prime \prime}}^{1}\right)$ is a conflation if 
and only if the corresponding sequences $0 \rightarrow P^{\prime i} \rightarrow P^{i} \rightarrow P^{\prime \prime i} \rightarrow 0$ of $R$-modules are short exact, $i=0,1$. Moreover, with this exact structure $\mathrm{MF}_{R}(f)$ is a Frobenius category, and its projective objects are equal to direct summands of an object of the form $\left(\operatorname{Id}_{P}, f \operatorname{Id}_{P}\right) \oplus\left(f \operatorname{Id}_{P}, \operatorname{Id}_{P}\right)$ for a projective $R$-module $P$; compare [16, Chapter I, 3.2] and [19, Example 5.3].

Denote by $\mathcal{F}$ the full subcategory of $\mathrm{MF}_{R}(f)$ consisting of objects of the form $\left(\operatorname{Id}_{P}, f \operatorname{Id}_{P}\right)$ for a projective $R$-module $P$. We claim that $\mathcal{F}$ satisfies the conditions in Theorem 3.1. Indeed, for a matrix factorization $\left(d_{P}^{0}, d_{P}^{1}\right)$, the following two sequences

$$
\left(d_{P}^{0}, d_{P}^{1}\right) \stackrel{\left(d_{P}^{0}, \operatorname{Id}_{P^{1}}\right)}{\longrightarrow}\left(\operatorname{Id}_{P^{1}}, f \operatorname{Id}_{P^{1}}\right) \longrightarrow(0,0)
$$

and

$$
(0,0) \rightarrow\left(\operatorname{Id}_{P^{0}}, f \operatorname{Id}_{P^{0}}\right) \stackrel{\left(\operatorname{Id}_{P^{0}}, d_{P}^{0}\right)}{\longrightarrow}\left(d_{P}^{0}, d_{P}^{1}\right)
$$

are the required sequences in (1) and (2), respectively. In this way, we get a factor Frobenius category $\mathrm{MF}_{R}(f) /[\mathcal{F}]$.

\section{Frobenius category and Cohen-Macaulay module}

In this section we will show that any Frobenius category is equivalent, as exact categories, to an admissible subcategory of the Frobenius category formed by CohenMacaulay modules over an additive category. This is an analogue of Gabriel-Quillen's embedding theorem for Frobenius categories; see [8, 18]. In particular, our result suggests that the category of Cohen-Macaulay modules serves as a standard model for Frobenius categories. We apply the obtained result to recover a part of [21, Theorem C]. We also make an application to the category of matrix factorizations.

Let $\mathcal{C}$ be an additive category. Denote by $\operatorname{Mod} \mathcal{C}$ the (large) abelian category of additive contravariant functors from $\mathcal{C}$ to the category of abelian groups; by abuse of terminology these functors are called $\mathcal{C}$-modules. Note that exact sequences of $\mathcal{C}$-modules are given by sequences of functors over $\mathcal{C}$, which are exact taking values at each object $C \in \mathcal{C}$.

For an object $C$ in $\mathcal{C}$, denote by $H_{C}=\operatorname{Hom}_{\mathcal{C}}(-, C)$ the corresponding representable functor. This gives rise to the Yoneda functor $H: \mathcal{C} \rightarrow \operatorname{Mod} \mathcal{C}$. Yoneda Lemma says that there exists a natural isomorphism $\operatorname{Hom}_{\operatorname{Mod}} \mathcal{C}\left(H_{C}, M\right) \simeq M(C)$ for each object $C \in \mathcal{C}$ and $M \in \operatorname{Mod} \mathcal{C}$. From these one infers that the Yoneda functor $H$ is fully faithful and the modules $H_{C}$ are projective for all $C \in \mathcal{C}$. Recall that a $\mathcal{C}$-module $M$ is finitely generated provided that there exists an epimorphism $H_{C} \rightarrow M$ for some object $C \in \mathcal{C}$. Observe that a $\mathcal{C}$-module is finitely generated projective if and only if it is a direct summand of $H_{C}$ for an object $C \in \mathcal{C}$. For details, we refer to 23 .

Recall that a cochain complex $P^{\bullet}=\left(P^{n}, d^{n}: P^{n} \rightarrow P^{n+1}\right)_{n \in \mathbb{Z}}$ consisting of finitely generated projective $\mathcal{C}$-modules is said to be totally acyclic provided that it is acyclic and for each object $C$ the Hom complex $\operatorname{Hom}_{\text {Mod } \mathcal{C}}\left(P^{\bullet}, H_{C}\right)$ is acyclic; compare [ 5 , p.400]. Following [7] and [6] a $\mathcal{C}$-module $M$ is said to be (maximal) Cohen-Macaulay provided that there exists a totally acyclic complex $P^{\bullet}$ such that the 0 -th cocycle $Z^{0}\left(P^{\bullet}\right)$ is isomorphic to $M$. In this case, the complex $P^{\bullet}$ is said to be a complete resolution of $M$. Observe that a finitely generated projective $\mathcal{C}$-module $P$ is CohenMacaulay, since we may take its complete resolution as $\cdots \rightarrow 0 \rightarrow P \stackrel{\text { Id } P}{\rightarrow} P \rightarrow 0 \rightarrow$ ... Note that in the literature, Cohen-Macaulay modules are also called modules of G-dimension zero ([1]) and Gorenstein-projective modules ([13]). Let us remark that Cohen-Macaulay modules are closely related to singularity categories $([7,24,9])$. 
Denote by $\operatorname{CM}(\mathcal{C})$ the full subcategory of $\operatorname{Mod} \mathcal{C}$ consisting of Cohen-Macaulay $\mathcal{C}$-modules. Note that since each Cohen-Macaulay module is finitely generated, the category $\operatorname{CM}(\mathcal{C})$ has small Hom sets. Observe that $\operatorname{CM}(\mathcal{C}) \subseteq \operatorname{Mod} \mathcal{C}$ is closed under extensions; compare [2, Propositon 5.1]. Then it becomes an exact category such that conflations are induced by short exact sequences with terms in $\operatorname{CM}(\mathcal{C})$.

The following result is well known; compare [9, Proposition 3.1(1)]. For the definition of an admissible subcategory, see Section 2.

Lemma 4.1. The exact category $\operatorname{CM}(\mathcal{C})$ is Frobenius; moreover, its projective objects are equal to finitely generated projective $\mathcal{C}$-modules. Consequently, any admissible subcategory of $\mathrm{CM}(\mathcal{C})$ is a Frobenius category.

Proof. Observe first that for a Cohen-Macaulay $\mathcal{C}$-module $M$ and a finitely generated projective $\mathcal{C}$-module $P$ we have $\operatorname{Ext}_{\text {Mod } \mathcal{C}}^{i}(M, P)=0$ for $i \geq 1$; compare [11, Lemma 2.1]. Hence the object $P$ is injective in $\operatorname{CM}(\mathcal{C})$; while it is clearly projective in $\operatorname{CM}(\mathcal{C})$. Observe from the definition that for each Cohen-Macaulay module $M$ with its complete resolution $P^{\bullet}$, we have two conflations $Z^{-1}\left(P^{\bullet}\right) \rightarrow P^{-1} \rightarrow M$ and $M \rightarrow P^{0} \rightarrow Z^{1}\left(P^{\bullet}\right)$. These two conflations imply that the exact category $\operatorname{CM}(\mathcal{C})$ has enough projective and enough injective objects; moreover, from these one infers that the class of projective objects coincides with the class of injective objects, both of which are equal to the class of finitely generated projective $\mathcal{C}$-modules. This shows that the category $\operatorname{CM}(\mathcal{C})$ is a Frobenius category, and the last statement follows immediately; see Section 2.

Let $(\mathcal{A}, \mathcal{E})$ be a Frobenius category. Denote by $\mathcal{P}$ the full subcategory of its projective objects. Consider the category $\operatorname{Mod} \mathcal{P}$ of $\mathcal{P}$-modules. For each object $A$ in $\mathcal{A}$ denote by $h_{A}$ the $\mathcal{P}$-module obtained by restricting the functor $H_{A}=\operatorname{Hom}_{\mathcal{A}}(-, A)$ on $\mathcal{P}$. This yields a functor $h: \mathcal{A} \rightarrow \operatorname{Mod} \mathcal{P}$ sending $A$ to $h_{A}$; such a functor is known as the restricted Yoneda functor. Observe that for an object $P \in \mathcal{P}$ we have $h_{P}=H_{P}$.

Recall from Lemma 4.1 that an admissible subcategory of the category of CohenMacaulay modules is Frobenius. In fact, all Frobenius categories arise in this way. This is our second result, which is an analogue of Gabriel-Quillen's embedding theorem for Frobenius categories; see [18, Proposition A.2] and [8, Theorem A.1].

Theorem 4.2. Use the notation as above. Then the restricted Yoneda functor $h: \mathcal{A} \rightarrow \operatorname{Mod} \mathcal{P}$ induces an equivalence of exact categories between $\mathcal{A}$ and an admissible subcategory of $\mathrm{CM}(\mathcal{P})$.

Proof. We will divide the proof into four steps. First observe that the functor $h$ sends conflations in $\mathcal{A}$ to short exact sequences of $\mathcal{P}$-modules, and sends projective objects in $\mathcal{A}$ to representable functors over $\mathcal{P}$, in particular, finitely generated projective $\mathcal{P}$-modules.

Step 1. We will show that for each object $A \in \mathcal{A}$ the $\mathcal{P}$-module $h_{A}$ is CohenMacaulay. For this, take conflations $\eta^{i}: A^{i} \rightarrow P^{i} \rightarrow A^{i+1}$ such that $A^{0}=A$ and $P^{i}$ 's are projective for $i \in \mathbb{Z}$. Applying $h$ to these conflations we get short exact sequences $0 \rightarrow h_{A^{i}} \rightarrow h_{P^{i}} \rightarrow h_{A^{i+1}} \rightarrow 0$. Splicing these short exact sequences we get an acyclic complex $h_{P}$ of finitely generated projective $\mathcal{P}$-modules which satisfies that $Z^{0}\left(h_{P} \bullet\right) \simeq h_{A}$. It remains to show that the complex $h_{P} \bullet$ satisfies that for each object $P \in \mathcal{P}$ the Hom complex $\operatorname{Hom}_{\mathrm{Mod}} \mathcal{P}\left(h_{P} \bullet, H_{P}\right)$ is acyclic. Here $H_{P}$ denotes the representable functor corresponding to $P$. Using Yoneda Lemma this Hom complex 
is isomorphic to the Hom complex $\operatorname{Hom}_{\mathcal{A}}\left(P^{\bullet}, P\right)$. Here the complex $P^{\bullet}$ in $\mathcal{A}$ is constructed by splicing the conflations $\eta^{i}$ together. Then the Hom complex $\operatorname{Hom}_{\mathcal{A}}\left(P^{\bullet}, P\right)$ is acyclic, since it is constructed by splicing the short exact sequence $\operatorname{Hom}_{\mathcal{P}}\left(\eta^{i}, P\right)$ together; here we use the fact that the object $P$ is injective in $\mathcal{A}$. Consequently the complex $h_{P}$ is totally acyclic and then the $\mathcal{P}$-module $h_{A}$ is Cohen-Macaulay.

Step 2. We will show that the functor $h$ is fully faithful. This is indeed fairly standard; compare the argument in [3, p.102]. We will only show the fullness, and by a similar argument one can show the faithfulness.

For an object $A \in \mathcal{A}$, we obtain from the conflations $\eta^{-1}$ and $\eta^{-2}$ in the first step a cokernel sequence $P^{-2} \rightarrow P^{-1} \rightarrow A \rightarrow 0$. This sequence induces a projective presentation $H_{P^{-2}} \rightarrow H_{P^{-1}} \rightarrow h_{A} \rightarrow 0$ of $\mathcal{P}$-modules. Similarly for another object $A^{\prime}$ we get a projective presentation $H_{P^{\prime-2}} \rightarrow H_{P^{\prime-1}} \rightarrow h_{A^{\prime}} \rightarrow 0$. Given a morphism $\theta: h_{A} \rightarrow h_{A^{\prime}}$, there exists a commutative diagram

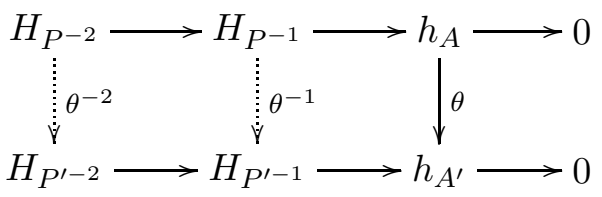

Observe that by Yoneda Lemma there exist morphisms $\mu^{-i}: P^{-i} \rightarrow P^{-i}$ such that $h_{\mu^{-i}}=\theta^{-i}$; moreover, these two morphisms make the left side square in the following diagram commute.

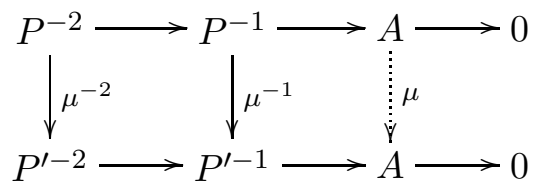

Since the two rows in the diagram above are cokernel sequences, one infers that there exists $\mu: A \rightarrow A^{\prime}$ making the diagram commute. It is direct to see that $h_{\mu}=\theta$ and this proves that the functor $h$ is full.

Step 3. Denote by $\operatorname{Im} h$ the essential image of the functor $h$. We have shown that $\operatorname{Im} h \subseteq \operatorname{CM}(\mathcal{P})$. We will now show that it is extension-closed. Note that the functor $h$ sends projective objects to projective modules, and sends conflations to short exact sequences. This will imply that $\operatorname{Im} h$ is an admissible subcategory of $\operatorname{CM}(\mathcal{P})$; see Section 2.

Take a conflation $h_{X} \rightarrow M \rightarrow h_{Y}$ in $\operatorname{CM}(\mathcal{P})$ with $X, Y \in \mathcal{A}$. We will show that $M$ lies in $\operatorname{Im} h$. For this, take a conflation $X \rightarrow Q \stackrel{d}{\rightarrow} X^{\prime}$ with $Q$ projective. Then we have the following commutative exact diagram

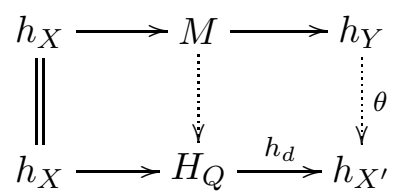

Here we use that $h_{Q}=H_{Q}$ is injective in $\operatorname{CM}(\mathcal{P})$; see Lemma 4.1. From this diagram we have a conflation $M \rightarrow h_{Y} \oplus H_{Q} \stackrel{\left(\theta, h_{d}\right)}{\rightarrow} h_{X}^{\prime}$ in $\mathrm{CM}(\mathcal{P})$. By the second step there exists a morphism $\mu: Y \rightarrow X^{\prime}$ such that $h_{\mu}=\theta$. By Lemma 2.1 there exists a conflation $Z \rightarrow Y \oplus Q \stackrel{(\mu, d)}{\rightarrow} X^{\prime}$ in $\mathcal{A}$ for some object $Z$. Applying $h$ to this conflation we get an isomorphism $M \simeq h_{Z}$. 
Step 4. We will show that the functor $h$ induces an equivalence of exact categories between $\mathcal{A}$ and $\operatorname{Im} h$. Then we are done with the proof. What remains to show is that the functor $h$ reflects exactness, that is, any sequence $\eta: X \stackrel{i}{\rightarrow} Y \stackrel{d}{\rightarrow} Z$ in $\mathcal{A}$ is a conflation provided that $h_{\eta}$ is a conflation in $\operatorname{Im} h$. View the functor $h$ as a full embedding. Since $h_{i}=\operatorname{Ker} h_{d}$, we have $i=\operatorname{Ker} d$. For each projective object $P$, we have an isomorphism $\operatorname{Hom}_{\mathcal{A}}(P, \eta) \simeq \operatorname{Hom}_{\mathrm{CM}(\mathcal{P})}\left(H_{P}, h_{\eta}\right)$ of sequences, and hence they are both exact. In particular, for a chosen deflation $P \stackrel{d^{\prime}}{\rightarrow} Z$ with $P$ projective, there exists a morphism $t: P \rightarrow Y$ such that $d \circ t=d^{\prime}$. Now we apply Lemma 2.2 to the morphism $d$. Then $d$ is a deflation and as its kernel, $i$ is an inflation. Consequently, the sequence $\eta$ is a conflation in $\mathcal{A}$, completing the proof.

We will apply Theorem 4.2 to recover a part of [21, Theorem C], which gives a surprising link between the category of vector bundles on weighted projective lines and a certain submodule category.

Example 4.3. Consider the factor Frobenius category $\mathcal{A}^{\prime}=$ vect $\mathbb{X} /[\mathcal{F}]$ in Example 3.3. The full subcategory $\mathcal{P}^{\prime}$ consisting of projective objects is equal to $\mathcal{P} /[\mathcal{F}]$. Then Theorem 4.2 implies that the associated restricted Yoneda functor $h: \mathcal{A}^{\prime} \rightarrow \operatorname{Mod} \mathcal{P}^{\prime}$ induces an equivalence of exact categories between $\mathcal{A}^{\prime}$ with an admissible subcategory of $\operatorname{CM}\left(\mathcal{P}^{\prime}\right)$. This might be viewed as a part of [21, Theorem $\left.\mathrm{C}\right]$.

In this situation, a highly nontrivial result is that the corresponding admissible subcategory is $\operatorname{CM}\left(\mathcal{P}^{\prime}\right)$ itself; compare [21, Propositon 3.18]. Finally observe that the category $\operatorname{CM}\left(\mathcal{P}^{\prime}\right)$ of Cohen-Macaulay $\mathcal{P}^{\prime}$-modules is equal to the submodule category $\mathcal{S}(\widetilde{p})$ of Ringel-Schmidmeier (by combining [21, Lemma B] and a graded version of [10, Lemma 4.3]). From these we conclude that there is an equivalence of exact categories between the factor category vect $\mathbb{X} /[\mathcal{F}]$ and the category $\mathcal{S}(\widetilde{p})$ of Ringel-Schmidmeier; this is [21, Theorem C].

In the next example, we apply Theorem 4.2 to the factor Frobenius category obtained in Example 3.4.

Example 4.4. Let $R$ be a commutative noetherian ring and let $f \in R$ be a regular element. We consider the factor Frobenius category $\mathcal{A}=\mathrm{MF}_{R}(f) /[\mathcal{F}]$ in Example 3.4. Observe that its full subcategory $\mathcal{P}$ of projective objects is the additive closure of the object $T:=\left(f \operatorname{Id}_{R}, \operatorname{Id}_{R}\right)$; moreover, the endomorphism ring of $T$ (in $\mathcal{A}$ ) is isomorphic to the quotient ring $S:=R /(f)$. By a version of Morita equivalence we have an equivalence $\operatorname{Mod} \mathcal{P} \simeq \operatorname{Mod} S$ of module categories; here $\operatorname{Mod} S$ denotes the category of $S$-modules. Furthermore, this equivalence restricts to an equivalence $\mathrm{CM}(\mathcal{P}) \simeq \operatorname{CM}(S)$. Here, $\operatorname{CM}(S)$ is the category of (maximal) Cohen-Macaulay $S$ modules $([\mathbb{8}, 6])$. Together with this equivalence we apply Theorem 4.2 to $\mathcal{A}$. Then the restricted Yoneda functor

$$
h: \mathrm{MF}_{R}(f) /[\mathcal{F}] \longrightarrow \mathrm{CM}(S)
$$

identifies $\mathrm{MF}_{R}(f) /[\mathcal{F}]$ as an admissible subcategory of $\mathrm{CM}(S)$. We will describe this admissible subcategory of $\operatorname{CM}(S)$. For this end, we will first give another description of the functor $h$.

Consider the following functor

$$
\text { Cok: } \operatorname{MF}_{R}(f) \longrightarrow \mathrm{CM}(S)
$$

which sends a matrix factorization $\left(d_{P}^{0}, d_{P}^{1}\right)$ to Cok $d_{P}^{1}$ and which acts on morphisms naturally. Observe that Cok $d_{P}^{1}$ is indeed a Cohen-Macaulay $S$-module; compare [12, 
Proposition 5.1]. Note that the functor Cok is exact and vanishes on $\mathcal{F}$. Then we have an induced functor Cok: $\mathrm{MF}_{R}(f) /[\mathcal{F}] \rightarrow \mathrm{CM}(S)$. We claim that there is a natural isomorphism between $h$ and Cok. In fact, to see this isomorphism, it suffices to note the natural isomorphisms $\operatorname{Hom}_{\mathcal{A}}\left(T,\left(d_{P}^{0}, d_{P}^{1}\right)\right) \simeq \operatorname{Cok} d_{P}^{1}$ for all matrix factorizations $\left(d_{P}^{0}, d_{P}^{1}\right)$.

Denote by $\mathcal{B}$ the full subcategory of $\operatorname{CM}(S)$ consisting of modules which, when viewed as $R$-modules, have projective dimension at most one. Observe that $\mathcal{B}$ is an extension-closed exact subcategory of $\operatorname{CM}(S)$. Recall that in a matrix factorization $\left(d_{P}^{0}, d_{P}^{1}\right)$ both morphisms $d_{P}^{0}$ and $d_{P}^{1}$ are mono. It follows that the image of the functor Cok lies in $\mathcal{B}$. We claim that any module in $\mathcal{B}$ lies in the image of the functor Cok. To see this, for an $S$-module $M$ in $\mathcal{B}$ we take an exact sequence $0 \rightarrow P^{1} \stackrel{d_{P}^{1}}{\rightarrow} P^{0} \stackrel{\pi}{\rightarrow} M \rightarrow 0$ such that $P^{i}$ are finitely generated projective $R$-modules, $i=0,1$. Since $f$ vanishes on $M$, then $\pi \circ f \operatorname{Id}_{P^{0}}=0$ and then $f \operatorname{Id}_{P^{0}}$ factors uniquely through $d_{P}^{1}$. In this way, we obtain a morphism $d_{P}^{0}: P^{0} \rightarrow P^{1}$ such that $\left(d_{P}^{0}, d_{P}^{1}\right)$ is a matrix factorization. Observe that $M \simeq$ Cok $d_{P}^{1}$. This shows the claim. Recall that the two functors $h$ and Cok are isomorphic. Then we conclude that the essential image of $h$ is $\mathcal{B}$. In particular, the subcategory $\mathcal{B} \subseteq \mathrm{CM}(S)$ is admissible. Hence the restricted Yoneda functor induces an equivalence of exact categories $\mathrm{MF}_{R}(f) /[\mathcal{F}] \simeq \mathcal{B}$. One might compare this with [12, Corollary 6.3] and [24, Theorem 3.9].

The situation is particularly nice if we assume that the ring $R$ is regular, that is, $R$ has finite global dimension. In this case, the quotient ring $S$ is Gorenstein. Observe that each Cohen-Macaulay $S$-module has projective dimension at most one, when viewed as an $R$-module (by [22, Lemma 18.2(i)]), that is, $\mathcal{B}=\operatorname{CM}(S)$. Then the restricted Yoneda functor $h$ induces an equivalence of exact categories $\operatorname{MF}_{R}(f) /[\mathcal{F}] \simeq$ $\mathrm{CM}(S)$.

We introduce the following notion: a Frobenius category $\mathcal{A}$ is standard provided that the associated restricted Yoneda functor $h: \mathcal{A} \rightarrow \mathrm{CM}(\mathcal{P})$ is an equivalence of exact categories; this is equivalent by Theorem 4.2 to that the functor $h$ is dense. For example, one can show that a Frobenius abelian category is standard; Example 4.3 claims that the factor Frobenius category $\mathcal{A}^{\prime}$ is standard; Example 4.4 implies that for a regular ring $R$ and a regular element $f \in R$, the factor Frobenius category $\mathrm{MF}_{R}(f) /[\mathcal{F}]$ is standard.

In general, it would be very nice to have an intrinsic criterion on when a Frobenius category is standard.

\section{Frobenius CATEGory From EXACT CATEgory}

In this section we give sufficient conditions such that on an exact category with enough projective and enough injective objects there exists another natural exact structure, with which the given category becomes Frobenius. We apply the result to the morphism category of a Frobenius abelian category; it turns out that this morphism category has a natural Frobenius exact structure. This observation allows us to interpret the minimal monomorphism operation in [26] as a triangle functor, which is right adjoint to an inclusion triangle functor.

Let $(\mathcal{A}, \mathcal{E})$ be an exact category with enough projective and enough injective objects. We denote by $\mathcal{P}$ and $\mathcal{I}$ the full subcategory of $\mathcal{A}$ consisting of projective and 
injective objects, respectively. Note that the exact category $\mathcal{A}$ might not be Frobenius. The aim is to show that under certain conditions there is a new exact structure $\mathcal{E}^{\prime}$ on $\mathcal{A}$ such that $\left(\mathcal{A}, \mathcal{E}^{\prime}\right)$ is a Frobenius category.

Recall that a full additive subcategory $\mathcal{S}$ of $\mathcal{A}$ is said to be contravariantly finite provided that each object in $\mathcal{A}$ has a right $\mathcal{S}$-approximation. Dually one has the notion of covariantly finite subcategory ([4, Section 2]). For two full subcategories $\mathcal{X}$ and $\mathcal{Y}$ of $\mathcal{A}$, denote by $\mathcal{X} \vee \mathcal{Y}$ the smallest full additive subcategory of $\mathcal{A}$ which contains $\mathcal{X}$ and $\mathcal{Y}$ and is closed under taking direct summands.

Recall that for a full additive subcategory $\mathcal{S}$ of an exact category $\mathcal{A}$, a conflation $\eta: X \rightarrow Y \rightarrow Z$ is right $\mathcal{S}$-acyclic provided that the sequences $\operatorname{Hom}_{\mathcal{A}}(S, \eta)$ are short exact for all $S \in \mathcal{S}$. Dually one has the notion of left $\mathcal{S}$-acyclic conflation.

Here is our third result, which gives sufficient conditions such that there is a natural (and new) exact structure on $\mathcal{A}$, with which $\mathcal{A}$ becomes a Frobenius category.

Theorem 5.1. Use the notation as above. Assume that $\mathcal{P}^{\prime} \subseteq \mathcal{P}$ and $\mathcal{I}^{\prime} \subseteq \mathcal{I}$ are two full additive subcategories subject to the following conditions:

(1) $\mathcal{P}^{\prime} \vee \mathcal{I}=\mathcal{I}^{\prime} \vee \mathcal{P}$

(2) $\mathcal{P}^{\prime} \subseteq \mathcal{A}$ is covariantly finite and $\mathcal{I}^{\prime} \subseteq \mathcal{A}$ is contravariantly finite;

(3) the class of right $\mathcal{I}^{\prime}$-acyclic conflations coincides with the class of left $\mathcal{P}^{\prime}$ acyclic conflations.

Denote the class of conflations in (3) by $\mathcal{E}^{\prime}$. Then the pair $\left(\mathcal{A}, \mathcal{E}^{\prime}\right)$ is a Frobenius exact category.

The proof of this result is quite direct, once we notice the following general observation.

Lemma 5.2. Let $(\mathcal{A}, \mathcal{E})$ be an exact category. For a full additive subcategory $\mathcal{S} \subseteq \mathcal{A}$, denote by $\mathcal{E}^{\prime}$ the class of right $\mathcal{S}$-acyclic conflations. Then the pair $\left(\mathcal{A}, \mathcal{E}^{\prime}\right)$ is an exact category.

Proof. For a conflation $(i, d)$ in $\mathcal{E}^{\prime}$, we will temporarily call $i$ an $\mathcal{E}^{\prime}$-inflation and $d$ an $\mathcal{E}^{\prime}$-deflation. We verify the axioms for the pair $\left(\mathcal{A}, \mathcal{E}^{\prime}\right)$. Recall that the axiom $(\mathrm{Ex} 1)^{\mathrm{op}}$ can be deduced from the others; see [18, Appendix A]. So we only show the remaining four axioms. The axiom (Ex0) is clear. Recall that a deflation $d: Y \rightarrow Z$ is an $\mathcal{E}^{\prime}$-deflation if and only if every morphism from an object in $\mathcal{S}$ to $Z$ factors through $d$. This observation yields (Ex1) immediately.

Consider the pullback diagram in the axiom (Ex2); see Section 2. Assume that $d: Y \rightarrow Z$ is an $\mathcal{E}^{\prime}$-deflation. We will show that $d^{\prime}: Y^{\prime} \rightarrow Z^{\prime}$ is also an $\mathcal{E}^{\prime}$-deflation. Take a morphism $s: S \rightarrow Z^{\prime}$ with $S \in \mathcal{S}$. Since $d$ is an $\mathcal{E}^{\prime}$-deflation, the morphism $f \circ s$ lifts to $Y$, that is, there exists $s^{\prime}: S \rightarrow Y$ such that $d \circ s^{\prime}=f \circ s$. Using the universal property of the pullback diagram, we infer that there exists a unique morphism $t: S \rightarrow Y^{\prime}$ such that $d^{\prime} \circ t=s$ and $f^{\prime} \circ t=s^{\prime}$. In particular, the morphism $s$ factors through $d^{\prime}$, proving that $d^{\prime}$ is an $\mathcal{E}^{\prime}$-deflation.

It remains to verify $(\mathrm{Ex} 2)^{\mathrm{op}}$. Consider the pushout diagram in $(\mathrm{Ex} 2)^{\mathrm{op}}$; see Section 2. We assume that $i: X \rightarrow Y$ is an $\mathcal{E}^{\prime}$-inflation. We will show that $i^{\prime}$ is an $\mathcal{E}^{\prime}$-inflation. We apply the dual of Lemma 2.1 to get the following commutative diagram such that 
the two rows are conflations

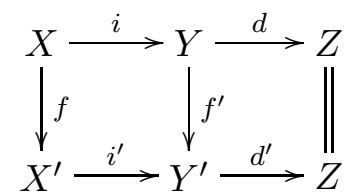

Here $d=d^{\prime} \circ f^{\prime}$. The fact that $i$ is an $\mathcal{E}^{\prime}$-inflation implies that $d$ is an $\mathcal{E}^{\prime}$-deflation. Consider any morphism $s: S \rightarrow Z$ with $S \in \mathcal{S}$. Then $s$ factors through $d$. Since $d=d^{\prime} \circ f^{\prime}$, we infer that the morphism $s$ factors through $d^{\prime}$. This shows that $d^{\prime}: Y^{\prime} \rightarrow Z$ is an $\mathcal{E}^{\prime}$-deflations and then $i^{\prime}$ is an $\mathcal{E}^{\prime}$-inflation. We are done.

Proof of Theorem 5.1; $\quad$ By Lemma 5.2 the pair $\left(\mathcal{A}, \mathcal{E}^{\prime}\right)$ is an exact category. We will call a conflation in $\mathcal{E}^{\prime}$ an $\mathcal{E}^{\prime}$-conflation. Note by the condition (3) that the objects in $\mathcal{P}^{\prime} \vee \mathcal{I}=\mathcal{I}^{\prime} \vee \mathcal{P}$ are projective and injective in the exact category $\left(\mathcal{A}, \mathcal{E}^{\prime}\right)$.

Observe from the condition (3) that a conflation $X \stackrel{i}{\rightarrow} Y \stackrel{d}{\rightarrow} Z$ is an $\mathcal{E}^{\prime}$-conflation if and only if any morphism from an object in $\mathcal{I}^{\prime}$ to $Z$ factors through $d$, if and only if any morphism from $X$ to an object in $\mathcal{P}^{\prime}$ factors through $i$. For an object $Z$ in $\mathcal{A}$, take a conflation $d: P \rightarrow Z$ with $P \in \mathcal{P}$ and a right $\mathcal{I}^{\prime}$-approximation $s: I^{\prime} \rightarrow Z$. By Lemma 2.1 the morphism $(d, s): P \oplus I^{\prime} \rightarrow Z$ is an deflation. It induces a conflation $\eta: X \rightarrow P \oplus I^{\prime} \stackrel{(d, s)}{\rightarrow} Z$. From the observation just made, we obtain that the conflation $\eta$ is an $\mathcal{E}^{\prime}$-conflation. This proves that the exact category $\left(\mathcal{A}, \mathcal{E}^{\prime}\right)$ has enough projective objects and the class of projective objects is equal to $\mathcal{I}^{\prime} \vee \mathcal{P}$. Dually one shows that the exact category $\left(\mathcal{A}, \mathcal{E}^{\prime}\right)$ has enough injective objects and the class of injective objects is equal to $\mathcal{P}^{\prime} \vee \mathcal{I}$. Then we conclude that the exact category $\left(\mathcal{A}, \mathcal{E}^{\prime}\right)$ is Frobenius, completing the proof.

We apply Theorem 5.1 to the morphism category of a Frobenius abelian category. This allows us to interpret the minimal monomorphism operation ([26]) as a right adjoint to an inclusion triangle functor.

Example 5.3. Let $\mathcal{A}$ be an abelian category. Denote by $\operatorname{Mor}(\mathcal{A})$ the morphism category of $\mathcal{A}$ : its objects are given by morphisms $\alpha: X \rightarrow Y$ in $\mathcal{A}$, and morphisms $(f, g): \alpha \rightarrow \alpha^{\prime}$ are given by commutative squares in $\mathcal{A}$, that is, two morphisms $f: X \rightarrow X^{\prime}$ and $g: Y \rightarrow Y^{\prime}$ such that $\alpha^{\prime} \circ f=g \circ \alpha$. It is an abelian category; a sequence $\alpha \rightarrow \alpha^{\prime} \rightarrow \alpha^{\prime \prime}$ in $\operatorname{Mor}(\mathcal{A})$ is exact if and only if the corresponding sequences of domains and targets are exact in $\mathcal{A}$; see [14, Corollary 1.2].

Assume that the abelian category $\mathcal{A}$ is Frobenius. In general the abelian category $\operatorname{Mor}(\mathcal{A})$ is not Frobenius. In fact, the category $\operatorname{Mor}(\mathcal{A})$ has enough projective and injective objects; projective objects are equal to objects of the form $(0 \rightarrow P) \oplus\left(Q \stackrel{\operatorname{Id}_{Q}}{\rightarrow}\right.$ $Q$ ) for some projective objects $P, Q \in \mathcal{A}$; dually injective objects are equal to objects of the form $(P \rightarrow 0) \oplus\left(Q \stackrel{\mathrm{Id}_{Q}}{\rightarrow} Q\right)$ for some injective objects $P, Q \in \mathcal{A}$; compare [26, Section 2]. Denote by $\mathcal{P}$ and $\mathcal{I}$ the full subcategory consisting of projective and injective objects in $\operatorname{Mor}(\mathcal{A})$, respectively.

Take $\mathcal{P}^{\prime} \subseteq \mathcal{P}$ to be the full subcategory consisting of objects of the form $0 \rightarrow P$. Take $\mathcal{I}^{\prime} \subseteq \mathcal{I}$ to be the full subcategory consisting of objects of the form $P \rightarrow 0$. We will verify the conditions in Theorem 5.1 .

The condition (1) is clear. To see (2), take an object $\alpha: X \rightarrow Y$ in $\operatorname{Mor}(\mathcal{A})$ and consider its cokernel $\pi: Y \rightarrow$ Cok $\alpha$ and a monomorphism $i$ : Cok $\alpha \rightarrow P$ with $P$ 
injective. Then the morphism $(0, i \circ \pi): \alpha \rightarrow(0 \rightarrow P)$ is a left $\mathcal{P}^{\prime}$-approximation. This proves that $\mathcal{P}^{\prime} \subseteq \operatorname{Mor}(\mathcal{A})$ is covariantly finite. Dually $\mathcal{I}^{\prime} \subseteq \operatorname{Mor}(\mathcal{A})$ is contravariantly finite. For (3), observe that a short exact sequence $0 \rightarrow \alpha \rightarrow \alpha^{\prime} \rightarrow \alpha^{\prime \prime} \rightarrow 0$ in $\operatorname{Mor}(\mathcal{A})$ is left $\mathcal{P}^{\prime}$-acyclic if and only if the corresponding sequence of cokernels is exact; by Snake Lemma this is equivalent to that the corresponding sequence of kernels is exact, and then equivalent to that the sequence is right $\mathcal{I}^{\prime}$-acyclic.

We apply Theorem 5.1 to obtain a Frobenius exact structure on $\operatorname{Mor}(\mathcal{A})$. Note that the corresponding conflations are given by short exact sequences in $\operatorname{Mor}(\mathcal{A})$ such that the associated sequences of kernels and cokernels are exact in $\mathcal{A}$; moreover, projective objects are equal to objects of the form $(0 \rightarrow P) \oplus\left(Q \stackrel{\operatorname{Id}_{Q}}{\rightarrow} Q\right) \oplus(R \rightarrow 0)$ for some projective objects $P, Q, R \in \mathcal{A}$. Denote by $\mathcal{P}_{\text {new }}$ the full subcategory of $\operatorname{Mor}(\mathcal{A})$ formed by these objects. We denote by $\underline{\operatorname{Mor}}(\mathcal{A})$ the stable category of $\operatorname{Mor}(\mathcal{A})$ modulo $\mathcal{P}_{\text {new }}$; it is a triangulated category $([16,18])$.

Recall that $\operatorname{Mon}(\mathcal{A})$ is the extension-closed exact subcategory of $\operatorname{Mor}(\mathcal{A})$ consisting of monomorphisms in $\mathcal{A}$; it is called the monomorphism category of $\mathcal{A}$. In fact, it is a Frobenius category such that its projective objects are equal to objects of the form $(0 \rightarrow P) \oplus\left(Q \stackrel{\operatorname{Id}_{Q}}{\rightarrow} Q\right)$ for projective objects $P, Q \in \mathcal{A}$. Denote by $\underline{\operatorname{Mon}}(\mathcal{A})$ the stable category. For details, see [10]. Hence we have an inclusion triangle functor inc: $\underline{\operatorname{Mon}}(\mathcal{A}) \hookrightarrow \underline{\operatorname{Mor}}(\mathcal{A})$. It is remarkable that this functor admits a right adjoint $\operatorname{inc}_{\rho}: \underline{\operatorname{Mor}}(\mathcal{A}) \rightarrow \underline{\operatorname{Mon}}(\mathcal{A})$ : for each object $\alpha: X \rightarrow Y$, consider a monomorphism $i_{X} \rightarrow I(X)$ such that $I(X)$ injective, and set $\operatorname{inc}_{\rho}(\alpha)=\left(\begin{array}{c}\alpha \\ i_{X}\end{array}\right): X \rightarrow Y \oplus I(X)$; the action of inc $_{\rho}$ on morphisms is defined naturally. In particular, the functor inc $\rho$ is a triangle functor; see [19, Section 8].

Suppose that the abelian category $\mathcal{A}$ has injective hulls. For an object $\alpha: X \rightarrow Y$ in $\operatorname{Mor}(\mathcal{A})$, consider its kernel $i: K \rightarrow X$ and an injective hull $j: K \rightarrow I(K)$. Then there exists a morphism $\bar{i}: X \rightarrow I(K)$ such that $\bar{i} \circ i=j$. Note that $\left(\frac{\alpha}{i}\right): X \rightarrow Y \oplus I(K)$ is a monomorphism; it is called the minimal monomorphism associated to $\alpha$ ([26, Sections 2,4]). Denote the minimal monomorphism by $\operatorname{Mimo}(\alpha)$. It is remarkable that there is a natural isomorphism between the object $\operatorname{inc}_{\rho}(\alpha)$ and $\operatorname{Mimo}(\alpha)$ in the stable category $\underline{\operatorname{Mon}}(\mathcal{A})$; compare [26, Section 4, Claim 2]. Then the minimal monomorphism operation Mimo(-) becomes naturally a triangle functor.

We would like to point out that the Frobenius category $\operatorname{Mor}(\mathcal{A})$ in the above example is standard, that is, the associated restricted Yoneda functor yields an equivalence $\operatorname{Mor}(\mathcal{A}) \simeq \operatorname{CM}\left(\mathcal{P}_{\text {new }}\right)$ of exact categories; see Section 4. Indeed, both exact categories are equivalent to the category of left exact sequences in $\mathcal{A}$ with the obvious exact structure. This observation and its generalization will be treated elsewhere.

\section{REFERENCES}

[1] M. Auslander And M. Bridger, Stable module category, Mem. Amer. Math. Soc. 94, 1969.

[2] M. Auslander and I. Reiten, Applications of contravariantly finite subcategories, Adv. Math. 86 (1991), 111-152.

[3] M. Auslander, I. Reiten, and S.O. Smalø, Representation Theory of Artin Algebras. Cambridge Studies in Adv. Math. 36, Cambridge Univ. Press, Cambridge, 1995.

[4] M. Auslander and S.O. Smalø, Almost split sequencrs in subcategories, J. Algebra 69 (1981), 426-454.

[5] L.L. Avramov and A. Martsinkovsky, Absolute, relative and Tate cohomology of modules of finite Gorenstein dimension, Proc. London Math. Soc. (3) 85 (2002), 393-440.

[6] A. Beligiannis, Cohen-Macaulay modules, (co)torsion pairs and virtually Gorenstein algebras, J. Algebra 288 (2005), 137-211. 
[7] R.O. Buchweitz, Maximal Cohen-Macaulay Modules and Tate Cohomology over Gorenstein Rings. Unpublished manuscript, 1987.

[8] T. Bühler, Exact categories, Expo. Math. 28 (2010), 1-69.

[9] X.W. Chen, Relative singularity categories and Gorenstein projective modules, Math. Nath., to appear; arXiv:0709.1762 2 .

[10] X.W. Chen, The stable monomorphism category of a Frobenius category, arXiv:0911.1987v2.

[11] L.W. Christensen, A. Frankild and H. Holm, On Gorenstein projective, injective and flat dimensions- a functorial description with applications, J. Algebra 302 (2006), 231-279.

[12] D. Eisenbud, Homological algebra on a complete intersection, with an application to group representations, Trans. Amer. Math. Soc. 260 (1) (1980), 35-64.

[13] E.E. Enochs, O.M.G. Jenda, Relative Homological Algebra. De Gruyter Expositions in Math. 30, Walter de Gruyter, Berlin, New York, 2000.

[14] R.M. Fossum, P. Griffith and I. Reiten, Trivial Extensions of Abelian Categories. Lecture Notes in Math. 456, Springer-Verlag, Berlin Heidelberg New York, 1975.

[15] W. Geigel And H. Lenzing, A class of weighted projective curves arising in representation theory of finite dimensional algebras, in: Singularities, representations of algebras and vector bundles, Lecture Notes in Math. 1273, 265-297, Springer, 1987.

[16] D. HApPEL, Triangulated Categories in the Representation Theory of Finite Dimensional Algebras. London Math. Soc. Lecture Notes Ser. 119, Cambridge Univ. Press, Cambridge, 1988.

[17] A. Heller, The loop-space functor in homological algebra, Trans. Amer. Math. Soc. 96 (1960), 382-394.

[18] B. Keller, Chain complexes and stable categories, Manuscripta Math. 67 (1990), 379-417.

[19] B. Keller, Derived categories and their uses, Handbook of Algebra 1, 671-701, NorthHolland, Amsterdam, 1996.

[20] D. Kussin, H. Lenzing And H. Meltzer, Triangle singularities, ADE-chains and weighted projective lines, preprint.

[21] D. Kussin, H. Lenzing And H. Meltzer, Nilpotent operators and weighted projective lines, arXiv: $1002.3797 \mathrm{v} 1$.

[22] H. Matsumura, Commuative Ring Theory. Cambridge Studies in Advanced Math. 8, Cambridge Univ. Press, Cambridge, 1986.

[23] B. Mitchell, Rings with several objects, Adv. Math. 8 (1972), 1-161.

[24] D. OrLOv, Triangulated categories of singularities and D-branes in Landau-Ginzburg models, Trudy Steklov Math. Institute 204 (2004), 240-262.

[25] D. QuiLLen, Higher algebraical K-theory I, Springer Lecture Notes in Math. 341, 1973, 85-147.

[26] C.M. Ringel And M. Schmidmeier, The Auslander-Reiten translation in submodule category, Trans. Amer. Math. Soc. 360 (2) (2008), 691-716.

[27] C.M. Ringel And M. Schmidmeier, Invariant subspaces of nilpotent linear operators, J. Reine Angew. Math. 614 (2008), 1-52.

Xiao-Wu Chen, Department of Mathematics, University of Science and Technology of China, Hefei 230026, P. R. China

Homepage: http://mail.ustc.edu.cn $/{ }^{\sim}$ xwchen

Current address: Institut fuer Mathematik, Universitaet Paderborn, 33095, Paderborn, Germany 